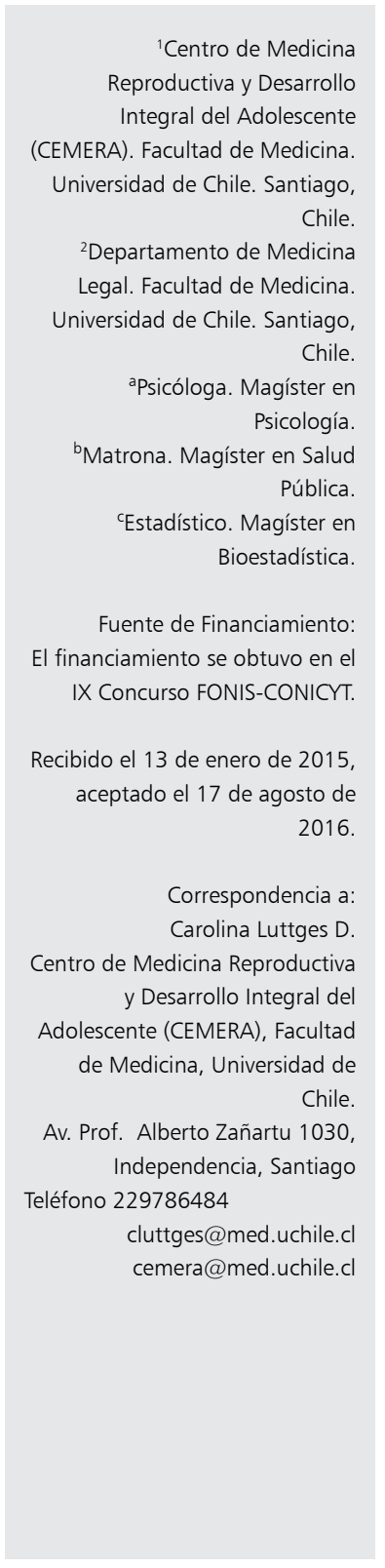

\section{Implicancias del marco legal chileno en la prevención del embarazo adolescente: conflicto e inseguridad en los profesionales de la salud}

\section{CAROLINA LUTTGES D. , $^{1, a}$ CAROLINA LEYTON M. ${ }^{1, a}$, INGRID LEAL F..$^{1, b}$, PAULINA TRONCOSO E. ${ }^{2}$, TEMÍSTOCLES MOLINA G. ${ }^{1, \mathrm{c}}$}

Background: Teenage pregnancy is a psychosocial and multifactorial problem described as a lack of exercise of rights in sexual and reproductive health. There are important aspects in the doctor-patient relationship and confidentiality that directly affect the continuity and quality of care. There are controversies in the laws relating to the provision of contraception and confidentiality, and those that protect the sexual indemnity, especially in adolescents under 14 years. Aim: To describe the implications of the legal framework for professional midwives in the care of adolescents younger than 14 years in sexual and reproductive health. Material and Methods: In-depth interviews were conducted to 13 female and 2 male midwives working at Primary Health Care Centers in the Metropolitan Region. Results: The attention of adolescents younger than 14 years in sexual and reproductive health involves medical-legal issues for health professionals. All professionals recognize that mandatory reporting sexual activity is a complex situation. All professionals notify pregnancies. In relation to the delivery of contraception, clinical care is problematic since professionals should take shelter from a legal standpoint. Conclusions: The medical-legal context of pregnant women under 14 years of age care generates a context of uncertainty and fear for professionals and becomes a source of conflict and insecurity in the exercise of the profession.

(Rev Med Chile 2016; 144: 1260-1265)

Key words: Health Personnel; Legislation, Medical; Pregnancy in Adolescence; Reproductive Rights.

4 n Chile, casi la mitad de los adolescentes son sexualmente activos y entre $14 \%$ y $17 \%$ de los adolescentes entre 13 y 15 años refiere haber iniciado relaciones sexuales. Una de las principales consecuencias del inicio de la actividad sexual no protegida es el embarazo adolescente, problema social asociado a la falta del ejercicio de los derechos sexuales y reproductivos como el acceso a la información y servicios de regulación de la fertilidad ${ }^{1-10}$.

Un estudio realizado en Chile sobre el seguimiento de la ley 20.418 "que fija las normas sobre información, orientación y prestaciones en materia de regulación de la fertilidad", refiere que una de las mayores preocupaciones de los adolescentes en la atención en salud sexual y reproductiva 
(SSR) es la confidencialidad, siendo una de las causas importantes por la cual los adolescentes no asisten a los centros de salud ${ }^{2}$. La confidencialidad es un aspecto medular en la relación entre el profesional de la salud y el paciente, por lo que tiene un impacto fundamental sobre el acceso, continuidad y calidad de los servicios de SSR para adolescentes $^{11-13}$.

Desde la perspectiva de los proveedores de salud, se mencionan diversas variables que afectan el acceso y entrega de una atención de salud de calidad a la población adolescente como la falta de capacitación y de regulaciones o guías que orienten a la atención de calidad de los y las adolescentes ${ }^{5,14}$. Se plantea además que cuando las leyes relativas a la SSR son nuevas y/o confusas, los proveedores no saben cómo afrontar temas sensibles como la confidencialidad y prescripción de anticonceptivos regulares y de emergencia ${ }^{5}$, lo que pudiera incidir en no otorgar un acceso oportuno a la atención o bien que sea negada ${ }^{15}$. Un estudio internacional refiere que las legislaciones relativas a la confidencialidad de la atención de adolescentes en SSR son vagas y confusas, y que el conocimiento que los prestadores de salud tienen sobre estas materias son insuficientes ${ }^{11}$. Aunque el derecho a la confidencialidad en SSR de los adolescentes está protegido por instrumentos jurídicos internacionales (derechos del niño, comité de derechos del niño y adolescentes en salud) ${ }^{13}$, en algunos países los marcos legislativos en materia de salud no han reconocido este derecho o lo han hecho de forma limitada.

En el caso de Chile, ha habido controversias en relación a las legislaciones relativas a la salud sexual y reproductiva y la confidencialidad. Existen leyes que aseguran la entrega de métodos anticonceptivos (MAC) regulares a adolescentes ${ }^{16}$ $\mathrm{y}$ al mismo tiempo, leyes que intentan proteger la indemnidad sexual de los y las adolescentes, estableciendo como violación toda actividad sexual en las menores de 14 años, aun cuando sea consentida y dentro de una relación afectiva con otro adolescente ${ }^{17,18}$. Este panorama legal ha generado confusión, desconocimiento y/o miedo en los equipos de salud sobre la atención a menores de 14 años en relación a la entrega de MAC y la confidencialidad, por las repercusiones que esto pueda ocasionar en sus carreras especialmente en las/os matronas/es ya que son estos profesionales quienes realizan estas prestaciones en la atención primaria de salud ${ }^{5,19-23}$.
El presente estudio tiene como objetivo conocer y describir las implicancias que el marco legal tiene para los profesionales matronas/es en la atención de adolescentes menores de 14 años en SSR.

\section{Material y Método}

Se realizó un estudio cualitativo y descriptivo, dado que se busca investigar, identificar, describir aspectos relevantes de un fenómeno poco conocido en la realidad nacional

Se utilizó un muestreo de casos homogéneos, de matronas/es de centros de atención primaria de salud (APS) de la Región Metropolitana. La selección de la muestra se realizó según los criterios de ser matronas y matrones de centros de atención primaria de salud, pertenecientes al área norte y sur oriente de la Región Metropolitana, dado que éstas cuentan con aquellas comunas con mayor proporción de nacidos vivos hijos de madres adolescentes $(19 \%-21,6 \%)^{3}$.

Se utilizó la entrevista en profundidad como técnica de producción de datos en base a guion temático semiestructurado abordando los tópicos de las prácticas clínicas y contexto legal en la atención de adolescentes para la prevención del embarazo.

Las entrevistas se realizaron en los respectivos lugares de trabajo, con duración aproximada de una hora y media y realizadas por los investigadores.

El análisis de los datos se realizó de acuerdo a la teoría fundamentada, a través de un análisis de contenido temático y levantamiento de categorías y subcategorías luego de un proceso de reducción progresiva de los datos emergentes ${ }^{24}$ (Tabla 1 ).

La muestra quedó conformada por 15 participantes (13 matronas y 2 matrones) de acuerdo al principio de la saturación de los datos. La mediana de edad correspondió a 36 años (rango 27-60). Los resultados se presentan apoyados de citas textuales provenientes de las entrevistas realizadas. La codificación indica primero que la entrevista (E) fue realizada a matrona o matrón $(\mathrm{M})$ y el número identifica al participante de acuerdo a la cronología en que se efectuaron las entrevistas.

Los aspectos éticos de esta investigación consideran el proceso de consentimiento informado, resguardando la confidencialidad de los participantes y la aprobación del comité de ética de la Facultad de Medicina de la Universidad de Chile (082-2012). 
Tabla 1. Categorías y subcategorías de respuesta de matronas y matrones de Atención Primaria de la Región Metropolitana

\begin{tabular}{|lll|}
\hline Metacategoría & Categorías & Subcategorías \\
Vivencias y significaciones de la & 1. Conflicto e inseguridad & - Denuncia \\
atención de menores de 14 años & & - Resguardo profesional \\
& 2. Relación profesional-usuario & - Confidencialidad \\
& & - Relación de confianza \\
\hline
\end{tabular}

\section{Resultados}

\section{Conflicto e inseguridad en el ejercicio de la profesión}

La atención de menores de 14 años involucra aspectos médico-legales en materia de la SSR que en los profesionales genera situaciones y toma de decisiones complejas en relación a la atención en este grupo etario. La legislación sobre abuso sexual ${ }^{17}$, ampliamente conocida por todos los profesionales entrevistados, genera una atención diferenciada en las adolescentes menores de 14 años, reconociendo que el criterio de límite de edad tiene directa relación con la normativa legal relacionada con el abuso sexual. Debido a la obligatoriedad de denunciar la actividad sexual en este grupo de adolescentes, la atención es compleja y se torna problemática cuando estas adolescentes no están embarazadas y solicitan algún MAC.

Este contexto médico-legal en la atención de menores de 14 años es para los profesionales una fuente de conflicto e inseguridad en el ejercicio de la profesión. Las matronas/nes entrevistados se sienten inseguros lo que de alguna forma coarta el accionar y el ejercicio del rol profesional en relación a la prevención del embarazo.

"pero por el otro lado que tiene que ver con la sospecha de abuso y todo ese tema, yo siento que ahí hay un vacío que en el fondo nos deja a nosotras súper desprotegidas" (EM4).

Existe variabilidad entre los profesionales sobre cómo proceder desde el punto de vista legal, cuando la adolescente solicita un MAC regular y da cuenta de tener actividad sexual consentida y no bajo coerción, situación constatada por el o la profesional. Si bien la mayoría de los profesionales no realiza una denuncia de la actividad sexual, buscan diversas formas para no judicializar la atención médica y resolver esta situación, pero manteniendo al mismo tiempo un resguardo pro- fesional ante el temor de alguna sanción de tipo legal. Las formas de resguardo pueden ser: solicitar la presencia de un adulto para dar la atención y prescribir el MAC regular; ampararse en el secreto profesional; registrar la atención como de otro tipo y así no explicitar la actividad sexual de la adolescente; o bien no entregar el MAC y buscar salidas alternativas, por ejemplo informarle que debe regresar cuando cumpla los 14 años o derivar a centro especializado de carácter privado.

"Antes venían sin adultos, pero y después de esa ley, tuvieron que estar viniendo con adultos responsables, que le firmaran y todo"

"Sí, me dio la tranquilidad y sentir que no estaba cometiendo un delito y que por último en última opción, tenía yo ese recurso por lo menos, resguardarme detrás de ese recurso que era el del secreto profesional"

“...un menor de 14 años viene a pedir anticonceptivo ahí nosotros no lo damos... ahora si viene una niñita de 13 años yo le hago consejería, le digo todo y apenas cumpla los 14 años, le voy a poner el implante o lo que sea"

"Nosotras las matronas, siempre hemos hablado, entonces nosotros en la ficha ponemos por ejemplo regulación de ciclo, no como anticonceptivo, pero son mañas que uno no debería ni ocupar"

Todos los profesionales realizan una denuncia o notificación de los embarazos, sin hacer distinciones si el embarazo es producto de una actividad sexual consentida o no. Es la matrona o matrón quien informa dentro de un contexto de consejería las razones legales de denunciar y sus posibles implicancias para la adolescente, y en la mayoría de los casos es el mismo profesional quien realiza la denuncia. Si bien la mayoría de los entrevistados reconocen el espíritu de protección del artículo 
de la ley sobre abuso sexual, se les produce un conflicto en relación a cumplir con la exigencia de denunciar, ya sea porque se considere el embarazo como un delito, verse involucrado en una instancia judicial, se vulnere la relación con la paciente y/o surja temor a represalias.

“...si teníamos una embarazada menor de 14 años, teníamos que llamar a la asistente social, para empezar a judicializar el caso..."

"... a mí a veces me dicen que no denuncie algunas cosas que hemos tenido que hacer, porque si te pasa algo..., porque uno, claro, tiene familia, viene a trabajar sola, sale del auto y no sabe qué va a pasar afuera"

\section{Relación profesional-usuario}

El aspecto médico-legal involucrado en la atención de menores de 14 años es una situación percibida por la mayoría de los entrevistados como un obstáculo para otorgar una atención adecuada y oportuna a las adolescentes menores que solicitan un MAC para prevenir un embarazo no deseado. El quiebre de la confidencialidad de la atención y el afectar la relación de confianza con la paciente es para la mayoría de los entrevistados un aspecto esencial en la atención de pacientes para la adherencia al MAC.

"te limita porque tú sabes que ahí había que hacer otra cosa, pero la ley te obliga a hacer lo que la ley dice y entonces uno siente que no es $100 \%$ eficiente, en lo que solicitó el adolescente..."

“... está esta parte de la ley [ley 19.927], pero también está mi rol como profesional, y mi ética...”

“...si uno sabe que la relación que está iniciando es con un adolescente también, a nosotros se nos complica...en relación a cómo cumplimos el marco legal, y cómo evitamos un embarazo, y es un conflicto para nosotras"

\section{"...también se infringe la confidencialidad que se da aquí con el paciente"}

La mayoría de los profesionales considera que debieran ser los equipos de salud debidamente capacitados quienes evalúen los casos de menores 14 años con actividad sexual y denunciar aquellos donde exista sospecha de una agresión sexual.

\section{Discusión}

El marco médico legal, relativo al límite de la edad de consentimiento sexual, se trasforma en un factor clave que enmarca la diferenciación del abordaje clínico y la vivencia de conflicto e inseguridad de los y las matronas en la atención para la prevención del embarazo en menores de 14 años.

Aun cuando reconocen la necesidad de atención en SSR de una adolescente menor de 14 años, se ven forzados a resguardar su trabajo por temor a las sanciones legales. Estos profesionales no cuentan con protocolos de acción formales provenientes de los organismos técnicos de la autoridad sanitaria, que den respaldo al quehacer profesional y seguridad en la práctica clínica, con el fin de evitar situaciones como el subregistro, el rechazo de la atención, condicionar la atención a la presencia de un adulto, entre otras. Estos aspectos generan un contexto de incertidumbre y trabajo inseguro, y conflicto en las y los profesionales por la tensión que se genera entre el deber reportar la actividad sexual consentida y el quiebre de la confidencialidad. Garantizar una atención confidencial es considerado como un aspecto esencial que facilita el acceso a los centros de salud, situación que no permite resolver la necesidad de atención en un grupo considerado de mayor vulnerabilidad y otorgar una atención de calidad que garantice el acceso oportuno al MAC.

En este sentido, las normativas sobre la edad de consentimiento sexual impactan sobre el acceso a los servicios de SSR, actuando como barreras en la medida que los profesionales de la salud las interpretan como un impedimento o dificultad legal para otorgar la atención. Desde el punto de vista de los profesionales, al mantener la confidencialidad en la atención de adolescentes menores de 14 años, podrían ser considerados como encubridores de un delito sexual infantil. Resultados similares, a nivel internacional, se han reportado en otros estudios sobre las barreras legales para el acceso de jóvenes y adolescentes a los servicios de SSR, en que los profesionales de la salud consideran que, desde un punto de vista penal, al otorgar la atención de forma confidencial, podrían ser vistos como facilitadores de la participación de adolescentes en actos sexuales indebidos ${ }^{25,26}$.

De acuerdo a los datos estadísticos nacionales, el inicio de actividad sexual antes de los 15 años ha ido aumentando en los últimos años, los que en su 
mayoría provienen de estratos socioeconómicos bajos, siendo por lo tanto beneficiarios del sistema público de atención primaria ${ }^{1-6}$. Tomando esto en consideración, podría inferirse que no se está resolviendo esta incipiente necesidad de salud, la que probablemente irá en aumento. El reporte de los participantes de este estudio hace necesario que los equipos de salud apliquen criterios que permitan evaluar caso a caso, discriminando entre los casos reales de abuso sexual de aquellos en que la actividad sexual es consentida y no abusiva, y abordarlos de manera integral y específica. La toma de decisiones en equipo, la capacitación y el respaldo institucional son factores que le dan al profesional una mayor confianza en el ejercicio profesional.

En casi todos los países se ha establecido legalmente un mínimo de edad para el consentimiento sexual. Según datos del Instituto Guttmacher, una proporción significativa de adolescentes ha tenido actividad sexual antes de la edad legal establecida en sus países, lo que significa un desafío para los profesionales y ministerios de salud ${ }^{27}$. Es necesaria una estrategia de abordaje integral en SSR para este grupo de adolescentes, que incluya capacitación específica en los aspectos técnicos y legales, el desarrollo de directrices formales de la autoridad sanitaria relativas a la atención, asegurar la confidencialidad y los derechos sexuales y reproductivos en el ámbito de la prevención del embarazo.

Este estudio tiene como limitación el que sus resultados no son necesariamente representativos ya que se centra en la realidad nacional del sistema público de atención y en comunas de ciertas características socioeconómicas y culturales, por lo que resulta un área que requiere de mayor desarrollo e investigación.

\section{Referencias}

1. Alt C, Aravena C, Argote L, Barretto M, Donoso A, Ferreiro JM, et al. Sexta Encuesta Nacional de Juventud. Santiago: Instituto Nacional de la Juventud; 2010. [Consultado el 23 de mayo de 2014]. Disponible en http://www.injuv.gob.cl/portal/wp-ontent/files_mf/ sextaencuestanacionaldejuventud.pdf

2. Dides C, Benavente C, Sáez I. Seguimiento a la Ley 20.418: Prevención del embarazo adolescente, educación sexual y anticoncepción de emergencia. Santiago: Minis- terio de salud y UNFPA; 2011. Plan Regional Andino de Prevención del Embarazo Adolescente [Consultado el 28 de mayo de 2014]. Disponible en http://www.icmer. org/documentos/adolescencia/te-educa-sexual-y-aechile\%20AMConcha\%2029.04.2013.pdf

3. Dides C, Benavente C, Moran JM. Diagnóstico de la situación del embarazo en la adolescencia en Chile. Santiago: 2008. Ministerio de Salud, FLACSO-Chile, UNFPA; 2008. Programa Género y Equidad.

4. Código penal, Ley 28.704 que Modifica artículos relativos a los delitos contra la libertad sexual y excluye a los sentenciados de los derechos de gracia, indulto y conmutación de la pena. Congreso de la República del Perú, (1303-2006) [Consultado el 8 de junio de 2014]. Disponible en http://www2.congreso.gob.pe/Sicr/TraDocEstProc/ TraDoc_condoc_2001.nsf/d99575da99ebfbe305256f2e006d1cf0/e164b0a268f4db600525745700688b4d/\$FILE/ SPL12494050413.pdf

5. Sadler M, Obach A, Luengo X, Biggs M. Estudio barreras de acceso a los servicios de salud para la prevención del embarazo adolescente en Chile. Santiago: Ministerio de Salud de Chile; 2011 [Consultado el 18 de noviembre de 2014]. Disponible en http://web.minsal.cl/portal/url/ item/ace74d077631463de04001011e011b94.pdf

6. Burdiles P, Santander R. Situación actual del embarazo adolescente en Chile. Santiago: Minsal; 2013. Programa Nacional de Salud Integral Adolescentes y Jóvenes. [Consultado el 22 de junio de 2014]. Disponible en http://web.minsal.cl/portal/url/item/d263acb5826c2826e04001016401271e.pdf

7. World Health Organization. Guidelines on Preventing Early Pregnancy and Poor Reproductive Outcomes Among Adolescents in Developing Countries; 2011. [Consultado el 22 de junio de 2015]. Disponible en http://www.who.int/immunization/hpv/target/preventing_early_pregnancy_and_poor_reproductive_outcomes_who_2006.pdf

8. Molina R, González E, Molina T. Madres niñas-adolescentes de 14 años y menos. Un grave problema de salud pública no resuelto en Chile. Rev Med Chile 2007; 135: 79-86.

9. Aravena A, Camelio F, Candia E, Cerda B, Chamorro MT, Leibovitz T, et al. Quinta Encuesta Nacional de Juventud. Santiago: Instituto Nacional de la Juventud; 2006. [Consultado el 23 de mayo de 2014]. Disponible en http://www.injuv.gob.cl/encuestasdejuventud/quintaencuestanacionaldejuventud.pdf

10. Aubry M, Barreto M, Acevedo J, Foster B, Lobos F. Séptima Encuesta Nacional de Juventud. Santiago: Instituto Nacional de la Juventud; 2012 [Consultado el 23 de mayo de 2014]. Disponible en http://www.injuv.gob. 
cl/portal/wp-content/files_mf/septimaencuestanacionaljuventud2.pdf

11. Lazarus J, Jaruseviciene L, Liljestrand J. Lithuanian general practitioner's knowledge of confidentiality laws in adolescent sexual and reproductive healthcare: A cross-sectional study. Scand J Public Health 2008; 36 (3): 303-9.

12. Naciones Unidas. Informe de la Conferencia Internacional sobre la Población y el Desarrollo El Cairo. Nueva York. Naciones Unidas, 1995. A/CONF.171/13/Rev.1.

13. Decreto 830, Promulga Convención Sobre Los Derechos Del Niño. Biblioteca del Congreso Nacional (20-111989) [Consultado el 6 de junio 2012]. Disponible en https://www.leychile.cl/N?i=15824\&f=1990-09-27\&p=

14. Departamento de Epidemiología MINSAL. Objetivos Sanitarios para la década 2000-2010. Boletín de vigilancia en Salud Pública de Chile 2002; 5: 1-12.

15. Castro R, Díaz S, Galán G, López C, Matamala M. Normas Nacionales sobre Regulación de la Fertilidad. Santiago: Ministerio de Salud; 2007.

16. Ley 20.418. Fija Normas sobre Información, Orientación y Prestaciones en Materia de Regulación de la Fertilidad. Biblioteca del Congreso Nacional, (28-01-2010) [Consultado el 5 de mayo de 2012]. Disponible en :http:// www.leychile.cl/ $\mathrm{N}$ ? $\mathrm{i}=1010482 \& \mathrm{f}=2010-02-02 \& \mathrm{p}=$

17. Código Penal y el Código Procesal Penal en materia de delitos de pornografía infantil. Ley número 19.927, Biblioteca del Congreso Nacional (14-01-2004) [Consultado el 22 de mayo de 2012]. Disponible en http:// www.leychile.cl/N?i=220055\&f=2004-01-14\&p=

18. Código de procedimiento penal y otros cuerpos legales en materias relativas al delito de violación. Ley 19.617. Biblioteca del Congreso Nacional, (12-07-1999) [Consultado el 22 de mayo de 2012]. Disponible en https:// www.leychile.cl/N?i=138814\&f=1999-09-17\&p=

19. Palma I. Trayectorias sexuales, preventivas y sociales en el embarazo no previsto de los segmentos juveniles en Chile. Revista Última Década 2010; 18 (33): 87-113.

20. Romero P, Oyarzún P. Anticoncepción en adolescen- tes ¿Qué dicen las leyes? Impacto de las leyes 19.927 y 20.418 en la Atención en salud de la adolescente al consultar por anticoncepción. Revista Sogia 2011; 18 (1): 8-16.

21. Hasen F. Documento de sistematización encuentros de adolescentes y salud, en salud los jóvenes eligen. Santiago: Minsal; 2011. [Consultado 18 de noviembre de 2014]. Disponible en http://web.minsal.cl/portal/url/ item/b554a9fa5044ef14e04001011e017f17.pdf

22. Estatuto Administrativo, Ley 18.834. Biblioteca del Congreso Nacional, (23-09-1989) [Consultado el 22 de mayo de 2012]. Disponible en http://bcn.cl/1v131

23. Código Penal de la República de Chile. Edición oficial del Código Penal aprobada por Decreto, No 417, (22-012010) [Consultado el 22 de mayo de 2012]. Disponible en http://bcn.cl/1uvs0

24. Strauss A, Corbin J. Bases de la investigación cualitativa: técnicas y procedimientos para desarrollar la teoría fundamentada. Editorial Universidad de Antioquia: 2002.

25. The International Planned Parenthood Federation. A multi-country study on legal barriers to young people's access to sexual and reproductive health services. El Salvador Case Study. London: IPPF; 2014 [Consultado el 26 de noviembre de 2015]. Disponible en http:// www.childrenslegalcentre.com/userfiles/file/ippf_coram_el_salvador_report_eng_web.pdf

26. Yarrow E, Anderson K, Apland K, Watson K. Can a restrictive law serve a protective purpose? The impact of age-restrictive laws on young people's access to sexual and reproductive health services. Reproductive Health Matters 2014; 22 (44): 148-56.

27. Anderson R, Panchaud C, Singh S, Watson K. Demystifying Data: A Guide to Using Evidence to Improve Young People's Sexual Health and Rights. New York: Guttmacher Institute; 2014 [Consultado el 02 de diciembre de 2015]. Disponible en https://www.guttmacher.org/sites/default/files/pdfs/pubs/demystifying-data. pdf 